\title{
Maternal and neonatal outcomes in COVID-19 infected pregnancies : a prospective cohort study
}

\author{
Abhilasha Parihar, Rupali Gupta, Ekta Chaudhary, Shweta Mishra, Manisha Agarwal, \\ Vishi Rawat, Priyanka Pandey, Anita Singh
}

Corresponding author: Dr. Shweta Mishra, Associate Professor, Dept. of Obs \& Gynae, Hind institute of medical sciences, Sitapur, UP, India; Email : shweta9mishra@gmail.com

Distributed under Attribution-Non Commercial - Share Alike 4.0 International (CC BY-NC-SA 4.0)

\begin{abstract}
Objective: To study maternal and neonatal outcome in COVID -19 positive pregnant women. Methodology: Prospective cohort study was done in a tertiary maternity unit in Hind Institute of Medical Sciences, level 2 COVID care centre. Data was analyzed for a cohort of 49 pregnant women tested positive for COVID-19 between 14/8/2020 and 20/10/2020 to assess the effect of COVID-19 on pregnancy and neonatal outcomes. Results: Among 49 women, 14 women $(28.57 \%)$ had mild symptoms, while 34 women $(69.38 \%)$ were asymptomatic, $1(2.04 \%)$ had moderate severity. 6 women had co-morbidities (12.24\%). 46 women underwent delivery and 2 women (4.08 \%) underwent abortion. Out of the 46 deliveries conducted $38(82.60 \%)$ underwent $\mathrm{C}$ - section, 1 (2.17 \%) had exploratory laparotomy and $7(15.21 \%)$ had normal delivery. Most frequent indication for performing C-section was fetal distress in 25 women (54.34\%). In per-operative findings of C-section conducted 20 women $(51.28 \%)$ were having meconium stained liquor. Other important per-operative finding was thinned out lower segment in all 11 pregnancies with previous scar, 5 women (12.8\%) had atonic PPH. There were two ICU (4.08\%) admissions and 1 maternal mortality (2.04\%). Amongst 46 delivered neonates, 2 were still born (4.34\%) 2 IUD (4.34\%), 13 preterm (28.26\%), 3 IUGR (6.52\%). 3 (6.52\%) neonates required NICU admissions for management. All babies were negative for COVID-19 after 5 days of delivery. Conclusion: In our study we found higher rates of $\mathrm{C}$-section in COVID positive pregnant women, severity of disease was not affected by pregnancy and there was no COVID associated mortality. Serocoversion to COVID negative status was $100 \%$ after 10 days. There was higher incidence of preterm, PROM, meconium stained liquor. We found no evidence of vertical transmission.
\end{abstract}

Keywords: COVID-19, pregnancy, antenatal, symptoms, C section, vertical transmission, mortality.

Humanity has witnessed its first ever worldwide pandemic of corona virus COVID-19, which originated in Wuhan, China, in December $2019^{1}$. Earlier similar corona virus infections have been reported like MERS (middle east respiratory syndrome) and SARS (severe acute respiratory syndrome) ${ }^{2}$ is caused by corona virus strain SARS COV $2^{3}$. India reported the first confirmed case of the COVID-19 on 30 January 2020 in Kerala state. By the end of December 2020 total number of cases in India were 1,02,66,674 with mortality of $1,48,738^{4}$.

Pregnancy is a state of altered immunity ${ }^{5}$ and COVID-19 is causing significant morbidity and mortality in dys- regulated immunological states like diabetes ${ }^{6,7}$. There is no previous data available of COVID-19 impact on pregnancy, however infection in pregnant with other strains of corona virus like SARS and MERS showed increased risk of preterm birth, stillbirth, miscarriage ${ }^{8,9}$. This pandemic brought changes in routine antenatal check up norms, because of various restrictions imposed, to curtail its spread. Guidelines are formulated and are continuously being upgraded on basis of variable presentations and course of disease. There has been marked impact on ANC (antenatal care) services because of limited number of ANC visits, only most essential and urgent investigations being done and

Received: $15^{\text {th }}$ January 2021, Peer review completed: $30^{\text {th }}$ March 2021, Accepted: $1^{\text {st }}$ May 2021.

Parihar A, Gupta R, Chaudhary E, Mishra S, Agarwal M, Rawat V, et al. Maternal and neonatal outcomes in COVID19 infected pregnancies : a prospective cohort study. The New Indian Journal of OBGYN. 2022; 8(2): 240 - 45. 
The New Indian Journal of OBGYN. 2021 (January-June);8(2)

physical presence being replaced by telemedicine ${ }^{10}$.

We have conducted our study on COVID-19 infected pregnant women. Our study aims to assess various maternal and neonatal outcomes that are directly or may be indirectly associated with COVID -19 infection in pregnant population. We require more such data to gear up and update our management thus having more preparedness for future. We hope findings of our study will contribute for the same.

\section{Materials and methods}

Total 49 COVID-19 positive pregnant women were admitted during duration of 2 months between 14/8/2020 and 20/10/2020, in maternity unit of COVID centre Hind Institute of Medical Sciences Barabanki, declared level 2 nodal centre by government. These were known COVID positive pregnant women with COVID antigen/RTPCR test positive, referred from other centers for admission in Hind hospital. We collected their prospective demographic and clinical information at time of admission as well as during their stay.

Inclusion criteria: All symptomatic/asymptomatic antenatal COVID-19 positive women referred and admitted to our centre by district authority.

Exclusion criteria: 1) All COVID-19 negative pregnancies, 2) All COVID-19 positive pregnancies requiring ventilator support/L3 facility.

Delivery and caesarean were conducted as per existing guidelines and protocols after routine ANC investigations. Babies were handed over to attendants and were isolated from mother till mother continued to be positive. RTPCR of newborns was sent on day 5 after delivery. Repeat RTPCR tests of women were sent at day 10 of their first test. Women were discharged if they were asymptomatic with negative report. They were continued admission on being symptomatic or with positive report. Mothers and babies were followed up via telephonic conversation up to 20 days of discharge.

Statistical analysis: In a Microsoft excel spreadsheet, the collected data was entered and evaluated, frequencies were expressed as percentages using tables, barchart and pie diagram to show variables distribution. Data was analysed regarding severity of symptoms, associated comorbidities, mode of delivery, indications of caesarean sections, significant preoperative findings, vertical transmission, neonatal intensive care unit admissions and any other adverse maternal and neonatal outcomes.

\section{Results}

In our hospital 49 antenatal COVID-19 positive women were admitted. Figure1 shows symptoms among these 49 women. 14 women (28.57\%) had mild symptoms (fever, cough, myalgia, anosmia) while 34 women $(69.38 \%)$ were asymptomatic. One women $(2.04 \%)$ had moderate severity and required high flow oxygen during $\mathrm{C}$-section (caesarean section) and on $1^{\text {st }}$ post operative day, same developed postpartum psychosis on $3^{\text {rd }}$ post operative day. None of the women developed serious respiratory symptoms during their course of stay and in follow up.

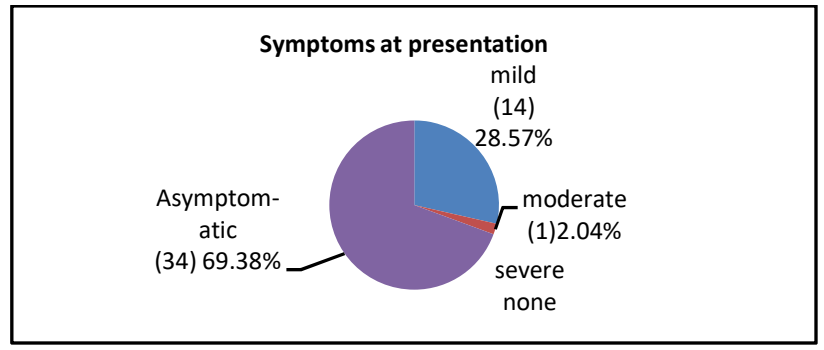

Figure 1: Symptoms at presentation

Among 49 admitted women 6 had co-morbidities (12.24\%) ( table 1). Co-morbidities included 1 case each of gestational diabetes, gestational hypertension, preeclampsia and HELLP syndrome (hemolysis, elevated liver enzyme, low platelets). Two women were of intrauterine death with multi-organ failure ( 1 case required dialysis).

46 women underwent delivery and 2 women (4.08\%)

\begin{tabular}{lll} 
Table 1: Maternal comorbidities $(\mathbf{n}=\mathbf{4 9})$ & & \\
\hline GDM & 1 & $2.04 \%$ \\
Gestational HTN & 1 & $2.04 \%$ \\
Preeclampsia & 1 & $2.04 \%$ \\
Sepsis with MOF & 2 & $4.08 \%$ \\
HELLP & 1 & $2.04 \%$ \\
Total & 6 & $12.24 \%$ \\
\hline
\end{tabular}

GDM - Gestational diabetes mellitus, HTN - Hypertension, MOF Multiple organ failure

underwent abortion (one was induced abortion for blighted ovum and other was spontaneous abortion at 10 weeks). 1 with hyperemesis gravidarum (16 week gestation) was conservatively managed. Table 2 shows maternal outcomes.

\begin{tabular}{llll} 
Table 2: Maternal outcomes $(\mathbf{n = 4 9 )}$ & & \\
\hline Modes of & Caesarian section & 38 & $82.60 \%$ \\
delivery & Normal vaginal delivery & 7 & $15.21 \%$ \\
$(\mathrm{n}=46)$ & Exploratory laparotomy & 1 & $2.17 \%$ \\
\hline Miscarriage & 2 & $4.08 \%$ \\
\hline ICU admission & 2 & $4.08 \%$ \\
\hline Mortality & 1 & $2.04 \%$ \\
\hline
\end{tabular}


Out of the 46 deliveries conducted 38 (82.60\%) underwent $\mathrm{C}$-section, 1 ( $2.17 \%)$ had exploratory laparotomy and $7(15.21 \%)$ underwent normal delivery including one VBAC (vaginal birth after caesarean) and one induced preterm delivery for IUD (intrauterine death) with MOF (multiple organ failure).

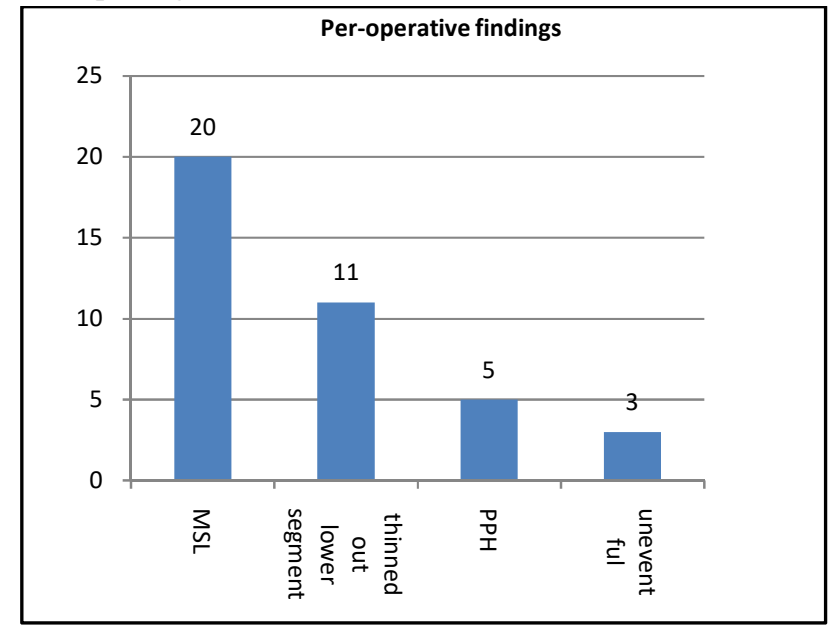

Most important and frequent indication for performing Csection was fetal distress (evident on non stress test) in 25 women (54.34\%). 11 women having fetal distress had premature rupture of membranes (PROM) that is $23.9 \%$ of total deliveries. Other indications were obstructed labor, CPD (cephalopelvic disproportion), cord prolapse, NPOL (non progress of labor), previous $\mathrm{C}$-section with scar tenderness. Figure 2 shows per-operative findings in $\mathrm{C}$ section/laparotomy of these patients. In per-operative findings 20 women $(51.28 \%)$ were having meconium stained liquor. While 18 women having meconium were in labor, 2 non laboring women taken up for emergency $\mathrm{C}$-section for fetal distress also had thick meconium. Other important peroperative finding was thinned out lower segment in all 11 women with previous scar though none of them were in advanced labor except the one case operated for previous Csection with ruptured uterus and bladder in $2^{\text {nd }}$ stage obstructed labor. Uterine tissue in most of the operated women was edematous, fragile and unhealthy specifically in women of previous scar. 5 women $(12.8 \%)$ had atonic PPH (post partum haemorrhage) which was medically and surgically managed, only 3 other operated cases had no such significant findings. There were 2 ICU (4.08\%) admissions among 49 women due to intrauterine demise with sepsis. One case was 29 week primi gravida with poor general condition having elevated liver function test, deranged kidney function test with preeclampsia. Induced vaginal delivery was done, subsequently postpartum liver and kidney profile improved. Other was primi gravida with term gestation with IUD and MOF taken for emergency C section for obstructed labor, patient was shifted to ICU postoperatively. She also underwent dialysis. Recovery period was prolonged with wound gaping and non healing bedsores. Post partum period was uneventful in most of the women however 1 woman reported prolonged anosmia, 1 had postpartum psychosis, in 2 operated cases there was wound gaping. Unfortunately one maternal mortality (2.04\%) happened in case of previous C - section with ruptured uterus and bladder however single live baby was rescued by exploratory laparotomy.

\begin{tabular}{lll} 
Table 3: Neonatal outcomes $(\mathbf{n = 4 6 )}$ & \\
\hline IUD & 2 & $4.34 \%$ \\
Still birth & 2 & $4.34 \%$ \\
Preterm & 13 & $28.26 \%$ \\
IUGR & 3 & $6.52 \%$ \\
NICU admission & 3 & $6.52 \%$ \\
RTPCR test negative after 5 days of live birth & 42 & $100 \%$ \\
\hline IUD - Intrauterine death, IUGR - Intrauterine growth restriction, NICU \\
- Neonatal intensive care unit, RT PCR - Reverse transcription
\end{tabular}
polymerase chain reaction

Amongst 46 delivered, 2 were still born (4.34 \%), 2 IUD (4.34\%), 13 preterm (28.26\%), 3 IUGR (intrauterine growth restriction) (6.52\%). $3(6.52 \%)$ neonates required NICU admissions for management (table 3). All neonates had normal Apgar score of 8 to 10, except 3 newborns who had low Apgar score of 3 to 4, they were resuscitated and admitted to NICU and subsequently were discharged in a healthy state. RTPCR tests of delivered babies were sent on day $5^{\text {th }}$ of delivery, all babies tested negative for COVID -19.

\section{Discussion}

In our study there were $69.38 \%$ asymptomatic patients and $28.57 \%$ having mild symptoms. Only one antenatal patient having moderate symptoms required oxygen support at the time of admission and during $\mathrm{C}$-section, same patient developed post partum psychosis on day 2 of operation rest all post-op patients were maintaining saturation and remained stable.

None of the patients deteriorated during hospital stay or during $\mathrm{C}$ - section /delivery. Follow up period was also uneventful indicating that pregnancy as such is not associated with severe consequences in COVID-19 infection. Our findings are similar to WHO joint mission report ${ }^{11}$ which investigated 147 pregnant women and suggested that pregnancy does not appear as a risk factor for severe COVID-19 disease, as opposed to pandemic influenza A (H1N1), however report concluded that having COVID-19 
during pregnancy may have an impact on fetal outcomes. In a study done by Arun Harishchandra Nayak et al on 144 pregnant women, $97 \%$ of women were asymptomatic or had mild symptoms ${ }^{12}$.

Our study suggested that SARS CoV-2 doesn't appear to have increased severity among pregnant women compared to normal population. In our study there was one maternal mortality which was related to obstructed labor with uterine and bladder rupture which in itself is a very high risk case. Study by Pradip Dashraath et al reported that COVID-19 outcomes for the mother appear more promising compared to those of SARS and MERS. Pooled data reveal a case fatality rate of $0 \%, 18 \%$, and $25 \%$ for COVID- 19 , SARS, and MERS, respectively; in the latter 2 disease syndromes, progressive respiratory failure and severe sepsis were the most frequent ${ }^{13}$.

In our study two ICU admissions can be attributed to multiple organ failure due to sepsis as a consequence of IUD. One patient required dialysis due to acute renal failure. Both women were high risk cases and their course of disease was more related with pregnancy related complications rather than COVID infection. However whether IUD was a consequence of COVID infection is still matter of research.

In our study among 46 deliveries $\mathrm{C}$ - section was required in 38 women $(82.60 \%)$. As per national family health survey rate of $\mathrm{C}$-section in Uttar Pradesh is $18.9 \%{ }^{14}$ indicating towards higher $\mathrm{C}$ - section rate among COVID-19 positive women. We performed $\mathrm{C}$-section for maternal or fetal indications and not due to COVID infection. Studies conducted on COVID positive pregnant women indicate towards higher $\mathrm{C}$ section rate among them. In a study by Liu $\mathrm{Y}$ et al there were $10 \mathrm{C}$-section in $13 \mathrm{COVID}$ positive pregnant women ${ }^{15}$. In a meta-analysis by Alvaro Francisco et al $65 \%$ women had $\mathrm{C}$ section and study by Lina Antouna et al also concluded higher $\mathrm{C}$ - section rates in COVID patients ${ }^{16,17}$. Arun Nayak et al concluded that $\mathrm{C}$-section rate were higher in COVID positive women as compared to non COVID but difference was not statistically significant ${ }^{12}$.

COVID pandemic has witnessed an era of tele-medicine, limited ANC visits and minimization of investigations and scans to essential and urgency based. Widespread fear and anxiety among pregnant further prevented them from visiting hospital. These factors may contribute to late detection of high risk women, incomplete workup eventually leading to higher rate of C- section when such women presented at term or in labor. Limited staff and difficulties faced by health care workers in protective gears like PPE kits may be a contributing factor in lowering threshold for $\mathrm{C}$ - section in
ANC. Meta-analysis conducted by Jerome Bouaziz et al concluded that only 11 women out of 174 COVID positive pregnancies had normal vaginal delivery rest all underwent C-section ${ }^{18}$. Most common indications for caesarean section were fetal distress and PROM. In our study also most common indication for C-section turned out to be fetal distress and 23.9\% women had PROM. Various studies have been conducted showing adverse fetal outcomes related to maternal mental status. In a study by Michael $\mathrm{T}$ Kinsella et al fetus of mothers with greater stress reported FHR variability therefore fetal distress may be linked to anxious mental status of COVID positive mothers ${ }^{19}$. However further research is required to assess various factors that may contribute to fetal distress in COVID infection. Study by Berrak Mizrak et al highlighted COVID pandemic has a significant potential for creating anxiety, adversity and fear, which has a negative emotional effect on pregnant people ${ }^{20}$. In our study one of the patient developed postpartum psychosis which may be related to underlying depression due to COVID infection.

In our per-operative findings, $51.28 \%$ had meconium stained liquor. In a study by Milind B Kamble on non COVID pregnant patients, out of 8765 deliveries incidence of MSAF was $13.9 \%$, as compared to this meconium in our group is quite high, reason needs further studies in this area ${ }^{21}$. Lower segment was found to be thinned out in all patients with previous scar and there was marked difficulty in suturing uterus because of fragile tissue. This may be attributed to inflammatory response of tissue to COVID-19 ${ }^{22}$. More studies are required on evidence of peroperative findings and complications in COVID positive women undergoing $\mathrm{C}$ - section for further correlation of our findings.

In our study there were $28.26 \%$ preterm deliveries which is more as compared to national rate of preterm births of $13 \%{ }^{23}$. This finding correlates with study by Oscar Martinez who found higher rate of PROM, preterm births and more NICU admissions in COVID positive group ${ }^{24}$. Similarly Lina Antouna suggested relatively higher rate of preterm birth, pre-eclampasia in COVID positive ANC ${ }^{17}$. Yangli Liu et al also found increased risk of perinatal complications like fetal distress, PPROM and still birth ${ }^{15}$. Study by David A Schwartz highlighted that MERS and SARS coronavirus had significant adverse clinical outcome including intensive care ventilatory support, IUGR, preterm ${ }^{9}$. Similar outcomes can be expected in COVID also. Systematic review done by Daniele Di Mascio et al quoted that preterm birth is the most common adverse pregnancy outcome in COVID and it was 
The New Indian Journal of OBGYN. 2021 (January-June);8(2)

also associated with higher rate of preeclampsia, caesarean, and perinatal death ${ }^{25}$. We found no evidence of vertical transmission in neonates. Also none developed symptoms in follow up. Huijun Chen et al reported no evidence of vertical transmission in 9 pregnant patients. Similarly Yang Liu et al suggested no evidence of vertical transmission in his study. In a meta-ananylsis conducted by Alvaro Francisco on 755 pregnant patients only $2 \%$ babies were tested positive for COVID with full recovery however author doesn't conclusively label it as vertical transmission. Same results were in study by Lina Antouna on 23 cohort of pregnant patients with no evidence of vertical transmission. Study conducted on 141 patients in India by Arun Harish Chandra Nayak suggested no evidence of vertical transmission ${ }^{15-17,26}$.

Study conducted by Chen et al on amniotic fluid, cord blood and neonatal throat swab samples was negative for SARS CoV-2 in 3 patients ${ }^{27}$. Hence there is no conclusive evidence of vertical transmission in COVID positive mothers and no evidence of neonatal infection. Temporary separation of newborn with mother can be observed to prevent exposure after birth till there are more reports on conclusive routes of transmission.

All patients were negative in repeat RTPCR COVID-19 done on $10^{\text {th }}$ day from symptoms onset showing good sero conversion rate among pregnancy.

\section{Conclusion}

Our study suggests that COVID-19 infection associated with pregnancy doesn't lead to increased severity or mortality. However there was increased rate of $\mathrm{C}$ section, fetal distress, PPROM, meconium stained liquor, preterm delivery. We found no evidence of vertical transmission. Our study is limited by small cohort size and small duration, more research is required in field of pregnancies affected by COVID-19 infection. Our study findings can contribute for further correlation.

Acknowledgment: Department of Obs \& Gynae, Hind Institute of Medical Sciences, Barabanki, UP.

\section{Conflict of interest: None. Disclaimer: Nil.}

\section{References}

1. Liu Y, Kuo R, Shih S. COVID-19: The first documented coronavirus pandemic in history. Biomedical Journal. 2020 Aug; 43(4): 328-33.

2. Hui DS. Epidemic and Emerging Coronaviruses (Severe Acute Respiratory Syndrome and Middle East Respiratory Syndrome). Clinics in Chest Medicine. 2017 Mar; 38(1): 71-86.
3. Koyama T, Platt D, Parida L. Variant analysis of SARSCoV-2 genomes. Bull World Health Organ. 2020 Jul 1; 98(7): 495-504.

4. Available from: https://mygov.in/corona-data/COVID19-statewise-status

5. Kourtis AP, Read JS, Jamieson DJ. Pregnancy and infection. N Engl J Med. 2014 Jun 5; 370(23): 2211-8.

6. Hodgson K, Morris J, Bridson T, Govan B, Rush C, Ketheesan N. Immunological mechanisms contributing to the double burden of diabetes and intracellular bacterial infections. Immunology. 2015 Feb;144(2): 171-85.

7. Guo W, Li M, Dong Y, Zhou H, Zhang Z, Tian C, et al. Diabetes is a risk factor for the progression and prognosis of COVID-19. Diabetes Metab Res Rev. 2020 Mar 31; e3319

8. Carlson A, Thung SF, Norwitz ER. H1N1 Influenza in Pregnancy: What All Obstetric Care Providers Ought to Know. Rev Obstet Gynecol. 2009; 2(3):139-45.

9. Schwartz DA, Graham AL. Potential Maternal and Infant Outcomes from (Wuhan) Coronavirus 2019 nCoV Infecting Pregnant Women: Lessons from SARS, MERS, and Other Human Coronavirus Infections. Viruses. 2020;12(2):E194.

10. ICMR - National Institute for Research in Reproductive Health. Guidance for Management of Pregnant Women in COVID-19 Pandemic. Mumbai; NIRRH; 2020. Available from: https:// www. icmr. gov. in/ pdf/covid/techdoc/Guidance_for_Management_of_Preg nant_Women_in_COVID19_Pandemic_12042020.pdf

11. World Health Organization report of the WHO-China joint mission on coronavirus Disease 2019 (COVID19). 2020. Available from: https://www.who.int/ docs/default-source/coronaviruse/who-china-jointmission-on-covid-19-final-report.pdf

12. Nayak AH, Kapote DS, Fonseca M, Chavan N, Mayekar $\mathrm{R}$, Sarmalkar $\mathrm{M}$, et al. Impact of the Coronavirus Infection in Pregnancy: A Preliminary Study of 141 Patients. J Obstet Gynaecol India. 2020 Aug; 70(4): 256-61.

13. Dashraath P, Wong JLJ, Lim MXK, Lim LM, Li S, Biswas A, et al. Coronavirus disease 2019 (COVID-19) pandemic and pregnancy. Am J Obstet Gynecol. 2020; 222(6): 521-31.

14. Available from: http://rchiips.org/nfhs/factsheet_nfhs4.shtmljog

15. Liu Y, Chen H, Tang K, Guo Y. Clinical manifestations and outcome of SARS-CoV-2 infection during 
The New Indian Journal of OBGYN. 2021 (January-June);8(2)

pregnancy. J Infect. 2020 Mar 5;S0163-4453(20) 30109-2

16. Lopes de Sousa ÁF, Carvalho HEF, Oliveira LB, Schneider G, Camargo ELS, Watanabe E, et al. Effects of COVID-19 Infection during Pregnancy and Neonatal Prognosis: What Is the Evidence. Int $\mathrm{J}$ Environ Res Public Health. 2020 Jun 11; 17(11): E4176.

17. Antoun L, Taweel NE, Ahmed I, Patni S, Honest H. Maternal COVID-19 infection, clinical characteristics, pregnancy, and neonatal outcome: A prospective cohort study. Eur J Obstet Gynecol Reprod Biol. 2020 Sep; 252: 559-62.

18. Bouaziz J, Even M, Isnard-Bogillot F, Vesale E, Nikpayam M, Mihalache A, et al. COVID-19 in pregnancy: What do we really know? F1000 Res. 2020 May 14; 9: 362.

19. Kinsella MT, Monk C. Impact of maternal stress, depression and anxiety on fetal neurobehavioral development. Clin Obstet Gynecol. 2009 Sep; 52(3): 425-40.

20. Mizrak Sahin B, Kabakci EN. The experiences of pregnant women during the COVID-19 pandemic in Turkey: A qualitative study. Women and Birth. 2021 Mar; 34(2):162-9.

21. Kamble MB, Jain P. Meconium aspiration syndrome: clinical profile, risk factors and outcome in central India. Int J Contemp Pediatr. 2018 Dec 24; 6(1):144.

22. Jose RJ, Manuel A. COVID-19 cytokine storm: the interplay between inflammation and coagulation. Lancet Respir Med. 2020 06;8(6):e46-e47.

23. Available from: https://www.healthynewbornnetwork. org/hnn- content/uploads/India-1.pdf
24. Spanish obstetric emergency group, Martinez Perez O, Rodriguez PP, Pardilla MBE, Perez N, Hernandez MRV, et al. The association between COVID-19 and preterm delivery: A cohort study with a multivariate analysis. BMC Pregnancy and Childbirth. 2021; 21: 273

25. Di Mascio D, Khalil A, Saccone G, Rizzo G, Buca D, Liberati $\mathrm{M}$, et al. Outcome of coronavirus spectrum infections (SARS, MERS, COVID-19) during pregnancy: a systematic review and meta-analysis. American Journal of Obstetrics \& Gynecology MFM. 2020 May; 2(2): 100107

26. Chen H, Guo J, Wang C, Luo F, Yu X, Zhang W, et al. Clinical characteristics and intrauterine vertical transmission potential of COVID-19 infection in nine pregnant women: a retrospective review of medical records. Lancet. 2020; 395(10226): 809 -15.

27. Chen S, Huang B, Luo DJ, Li X, Yang F, Zhao Y, et al. Pregnancy with new coronavirus infection: clinical characteristics and placental pathological analysis of three cases. Zhonghua Bing Li Xue Za Zhi. 2020 May 8; 49(5): 418-23.

\footnotetext{
Abhilasha Parihar ${ }^{1}$, Rupali Gupta ${ }^{2}$, Ekta Chaudhary ${ }^{3}$, Shweta Mishra ${ }^{4}$, Manisha Agarwal ${ }^{5}$, Vishi Rawat ${ }^{6}$, Priyanka Pandey ${ }^{7}$, Anita Singh ${ }^{8}$

${ }^{1}$ Assistant Professor; ${ }^{2}$ Assistant Professor; ${ }^{3}$ Associate Professor; ${ }^{4}$ Associate Professor; ${ }^{5}$ Professor \& HOD;

${ }^{6}$ Assistant Professor; ${ }^{7}$ Senior Resident; ${ }^{8}$ Senior Resident, Dept. of Obs \& Gynae, Hind institute of medical sciences, Sitapur, UP, India.
} 